\title{
Using recurrent neuro-fuzzy techniques for the identification and simulation of dynamic systems
}

\author{
Andreas Nürnberger ${ }^{\mathrm{a}, *}$, Arne Radetzky ${ }^{\mathrm{b}}$, Rudolf Kruse ${ }^{\mathrm{a}}$ \\ anstitute of Knowledge Processing and Language Engineering, Otto-Von-Guericke University of Magdeburg, \\ Universitätsplatz 2, D-39106 Magdeburg, Germany \\ ${ }^{\mathrm{b}}$ Institute of Applied Sciences in Medicine, ISM-Austria, Jakob Haringer Str. 3, A-5020 Salzburg, Austria
}

Received 27 July 1999; accepted 29 August 2000

\begin{abstract}
The identification and simulation of dynamic systems is still a challenging problem. In this article some basic aspects of neuro-fuzzy techniques for the identification and simulation of time-dependent physical systems are presented. In particular, a neuro-fuzzy model that can be used for the identification and the (real-time) simulation of viscoelastic models, is described. The presented model is motivated by a cooperative neuro-fuzzy approach based on a vectorized recurrent neural network architecture. The physical motivation of this model is illustrated and specific propagation procedures and a learning algorithm are presented. Moreover, the usability in practice is demonstrated by an application of the model in the area of surgical simulation. (C) 2001 Elsevier Science B.V. All rights reserved.

Keywords: System identification; Recurrent network; Neuro-fuzzy; Viscoelastic model; Virtual reality
\end{abstract}

\section{Motivation}

Nowadays the real-time simulation of deformable or elastic objects is necessary in many virtual reality applications. For example, in automobile industry water or fuel tubes have to be simulated to (interactively) optimise the design of the engine for maintenance and to develop virtual training courses for mechanics [42]. Another

\footnotetext{
* Corresponding author. Tel.: + 49-391-67-11358; fax: + 49-391-67-12018.

E-mail address: andreas.nuernberger@cs.uni-magdeburg.de (A. Nürnberger).
} 
growing field of interest is the design of virtual training environments for the education of ongoing surgeons and the development of virtual environments for computerassisted surgery [3]. In these areas it is very important to obtain visual convincing and physically acceptable simulations of the operation scenario [9]. Most of the already utilised methods for training and planning are based on static visualisation techniques. To improve the realism of virtual environments, a visual convincing static model of the scenario and the involved objects is not sufficient, because in many cases, objects can be deformed and modified at contact [38]. For the sake of interactivity and a low latency time, this has to be done in real-time with a graphic update frequency of at least 20 frames per second.

Unfortunately, a unified framework for the design of deformable models that can be used in virtual reality applications, is still missing. The reasons for this are mainly the occurring problems in the modelling phase (e.g. model structure selection and parameter determination) and the real-time restrictions during the simulation of the virtual objects.

\subsection{Modelling deformable virtual objects}

First of all, to obtain a deformable model of an object, an appropriate structure must be defined that complies with the specific demands of the application. These are, for example, the definition of an object model, which supports the modification of the object structure during real-time simulation (to perform cuts or ruptures), the simulation of object collisions or volume-preservation [33]. When a specific model structure has been selected, the respective model parameters must be defined or learned depending on the selected approach. Commonly used modelling approaches are differential equations [6], finite element models [1] and neural networks [15,35]. However, all existing approaches have specific insufficiencies. Differential equations require the definition of the physical parameters of the 'real' object, which are usually hard to determine. If measured data exist, e.g. time series data of the objects shape under influence of external forces, this leads to inverse, mostly ill-posed non-linear problems, which are still a field of mathematical research [13]. The design of finite element models is usually very time consuming, since an appropriate structure has to be defined [10]. The existing neural network based models on the other hand, need training data for learning, which is usually not available and has to be created, e.g. by use of a predefined finite-element model [14]. The learned networks are then used to replace the finite-element models and thus speed-up the real-time simulation.

\subsection{Real-time simulation of deformable virtual objects}

The computation of complex model descriptions is usually very time-consuming, thus, making their use in real-time applications impossible. Therefore, usually extensive model simplifications have to be done, which often lead to insufficient realism of the simulated object. A further problem is that even small or local changes of the model structure, e.g. for the simulation of cuts or ruptures, often require a rebuild of the whole model. Some approaches try to resolve these problems by pre-processing 
elementary deformations (see, for example, [8]). However, this is very time consuming for complex objects. The neural-network-based approaches usually did not support any modifications of the object structure, since they usually simulate complex parts of larger objects [14].

To resolve some of the problems mentioned above, in the following an approach based on a neuro-fuzzy system is presented, which defines a framework for modelling and the simulation of deformable virtual objects.

\section{Modelling and simulation using neuro-fuzzy techniques}

The proposed model was motivated by a combination of a fuzzy system and an artificial (recurrent) neural network, a so-called cooperative neuro-fuzzy system [24]. Neuro-fuzzy systems combine the capabilities of neural networks [35] (e.g. the ability to learn) and fuzzy systems [18] (e.g. interpretability). Here, the fuzzy system enables the definition of (initial) model parameters by use of prior expert knowledge. For example, terms like hard, very soft, low damping or very elastic can be used to define the (initial) model's behaviour. The neural network is used to optimise these parameters or learn all parameters if no suitable expert knowledge is available.

In the following a brief introduction to recurrent and vectorised neural networks is given and the principle ideas of model construction for the simulation and identification of dynamic systems and the combination with fuzzy systems are presented.

\subsection{Recurrent neural networks}

In a recurrent neural network (feedback network) self-loops and backward connections between nodes are allowed, which enables - in contrast to pure feed-forward architectures - the description of dynamic behaviour. The recurrence enables the network to 'remember' prior states of the propagation process and thus the network is able to use its prior states (and current input) to calculate its new state. Depending on the specific structure and weights it is possible to create networks that converge to fixed points, describe limited cycles or chaotic behaviour [19].

Formally, for a neuron $j$ the output $o_{j}(t)$ at time step $t$ is computed by use of the external input ext $(t-\mathrm{d} t)$, the output of connected neurons $o_{i}(t-\mathrm{d} t)$ and the network weights $w_{i j}$ :

$$
o_{j}(t)=f\left(\text { net }_{j}(t)\right)=f\left(\sum_{i} o_{i}(t-\mathrm{d} t) w_{i j}+\operatorname{ext}_{j}(t-\mathrm{d} t)\right) .
$$

For the continues case this leads to (see, for example [27]):

$$
\frac{\mathrm{d} o_{j}}{\mathrm{~d} t}=-o_{j}+f\left(\sum_{i} o_{i} w_{i j}+e x t_{j}\right) .
$$

Therefore, a recurrent network is capable of computing a system of differential equations and to learn its parameters. 
For a detailed discussion of recurrent neural networks see, for example, [15,19,27]. A discussion concerning hardware implementations of (recurrent) neural networks - which may be of interest to improve the real-time performance - and problem specific architectures, e.g. the use of recurrent neural networks to solve optimisation problems, can be found in [7].

\subsection{Vectorised (recurrent) neural networks}

A vectorisation of a (feed-forward or recurrent) neural network can be done by replacing the (scalar) processing elements of conventional networks (net input function net, transfer function $f$ and output function $o$ ) by vector-processing units and by vectorising the connections between the neurons. The weights of the network remain scalars. The propagation process in such networks can be done in the same way as in conventional (feed-forward or recurrent) neural networks. Also, existing learning procedures can be used to train them, since the principle structure, data flow and processing remain unchanged.

Vectorised networks can be transferred into conventional scalar networks if they do not use any specific vector operations. This can be done by creating a copy of each neuron and its connections - which must then share the same weight (even during learning) - for every dimension. Vectorised networks can be seen as a special neural network structure, or simply as a generic framework to be able to transfer neural network learning and propagation/simulation techniques to a different domain.

By use of a vectorised recurrent network, learning and simulation techniques can be transferred to a system of differential equations to learn its parameters (also known as solving an inverse problem [13]) and to compute it. Thus, a framework for a problem-specific modelling and definition process, and a problem-specific solution and propagation algorithm is given.

An example of a modified learning algorithm for a vectorised recurrent network - which will be referred in the description of the learning algorithm for the viscoelastic model - is presented in the following.

\subsubsection{Vectorised BPTT}

A frequently used learning method for recurrent neural networks is backpropagation through time (BPTT) [36,37]. The main idea of this method is to transfer the recurrent network into a feed-forward network by unfolding it in time [22]. Then, a conventional backpropagation learning method can be applied. The vectorised version of this algorithm can be described as follows.

The propagation function of a vectorised network can be defined as

$$
\vec{o}_{j}(t)=\vec{f}\left(n \vec{e} t_{j}(t)\right)=\vec{f}\left(\sum_{i} \vec{o}_{i}(t) w_{i j}+e \vec{x} t_{j}(t)\right)
$$

where $\vec{o}_{j}(t)$ is the output of neuron $j$ at time $t$ and $e \vec{x} t_{j}(t)$ the (external) input to the neuron (if any). 
Let $E$ be the total error, respectively, the cost function to be minimised, of all output neurons $\mathrm{k}$ over all time steps $t=0, \ldots, T$ :

$$
E=\sum_{t=0}^{T} E(t)=\sum_{t=0}^{T}\left[\frac{1}{2} \sum_{k}\left(\vec{E}_{k}(t)\right)^{2}\right]
$$

with

$$
\vec{E}_{k}(t)= \begin{cases}\vec{p}_{k}(t)-\vec{o}_{k}(t) & \text { if node } k \text { has a target, } \\ 0 & \text { output } \vec{p}_{k} \text { at time } t \\ \text { otherwise, }\end{cases}
$$

be an error measure for node $\mathrm{k}$. Then the error gradient can be derived as usual by defining

$$
\Delta w_{i j}=-\eta \frac{\partial E}{\partial w_{i j}}=-\eta \sum_{t=1}^{T} \frac{\partial E(t)}{\partial n \vec{e} t_{j}(t)} \frac{\partial n \vec{e} t_{j}(t)}{\partial w_{i j}}
$$

The error signal $\delta(t)$ for every neuron can be defined as

$$
\vec{\delta}_{j}(t)=-\frac{\partial E(t)}{\partial n \vec{e} t_{j}(t)}=-\frac{\partial E(t)}{\partial \vec{o}_{j}(t)} \frac{\partial \vec{o}_{j}(t)}{\partial n \vec{e} t_{j}(t)} .
$$

Thus, with

$$
\vec{A}_{j}(t)=\vec{E}_{j}(t)+\sum_{k} \vec{\delta}_{k}(t+1) w_{i k}
$$

the specific error signals can be obtained:

$$
\vec{\delta}_{j}(t)= \begin{cases}\vec{f}^{\prime}\left(n \vec{e} t_{j}(t)\right) \vec{E}_{j}(t) & \text { if } t=T, \\ \vec{f}^{\prime}\left(n \vec{e} t_{j}(t)\right) \vec{A}_{j}(t) & \text { if } 1 \leq t<T .\end{cases}
$$

With Eq. (2.6) the gradients can be derived after the propagation over all time steps:

$$
\Delta w_{i j}=-\eta \frac{\partial E}{\partial w_{i j}}=\eta \sum_{t=1}^{T} \vec{\delta}_{j}(t) \vec{o}_{i}(t-1) .
$$

It should be noted, that the only difference to the standard BPTT algorithm is the use of the scalar product in Eqs. (2.4), (2.9) and (2.10) Thus, for example, the error rate $\delta(t)$ 
depends on the vector component of the local error vector in direction to the vector of the local gradient of the transfer function $f$.

\subsection{Modelling a physical system by neural networks}

If a (physical) system has to be modelled by a neural network, first of all training patterns describing the input-output behaviour have to be determined. Then, a network - usually a feed-forward one - can be constructed. The number of input and output units has to match the number of input respectively output units of the physical system. The number of hidden units is mostly defined from experience or a pruning method is used $[35,15]$ Nevertheless, even if the obtained network has been successfully trained and pruned, the network structure is in many cases more complex than required. Furthermore, if some prior expert knowledge about the (physical) system is available, the network parameters cannot be initialised to simplify the training process. This may be necessary, if there are only a small number of training patterns available, as in many real world problems.

\subsubsection{Using prestructured networks}

Another method to model a physical system by use of a neural network is to construct a network as much as possible according to the mathematical model of the system. This seems to contradict the ideas of neural networks, since they were usually be seen as model-free architectures, but even in biological neural networks prestructured systems for specific purposes can be found (see, for example, [11]). The main advantages of this approach are the possibility to interpret the structure at any time, the possibility to initialise the network if prior knowledge is available - for example, supported by a fuzzy system as presented in the following - and the implicitly problem adjusted size of the network. Furthermore, the training process is usually simpler and faster and the approximation and generalisation quality is improved in contrast to common approaches. One disadvantage of this approach is that it is sometimes hard to transfer a mathematical model in a neural network structure, especially if differential equations have to be modelled by use of recurrent or vectorised architectures. Obviously, if no mathematical description of the system to be modelled is known, this approach cannot be used.

A simple example - the modelling of an integrator node - is presented in the following. Let us assume a simple recurrent neuron with one input and one output (Fig. 1).

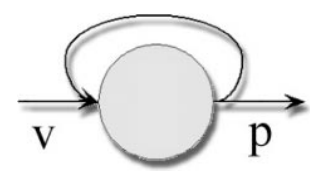

Fig. 1. A simple recurrent neuron. 
Let the transfer function $f:=i d, w_{p}:=1$ and $w_{v}:=\mathrm{d} t$. Then, the propagation function of this neuron is defined as

$$
\begin{aligned}
p(t) & =o(t)=f\left(\sum_{i} o_{i}(t-\mathrm{d} t) w_{i}+\operatorname{ext}_{j}(t-\mathrm{d} t)\right) \\
& =p(t-\mathrm{d} t) w_{\mathrm{p}}+v(t-\mathrm{d} t) w_{v} \\
& =p(t-\mathrm{d} t)+v(t-\mathrm{d} t) \mathrm{d} t
\end{aligned}
$$

By use of Eq. (2.12) it is obtained

$$
\frac{p(t)-p(t-\mathrm{d} t)}{\mathrm{d} t}=v(t-\mathrm{d} t)
$$

For $\mathrm{d} t \rightarrow 0$ this leads to

$$
\frac{\mathrm{d} p}{\mathrm{~d} t}=v(t)
$$

respectively

$$
p(t)=\int v(t) \mathrm{d} t .
$$

In this way even more complex differential equations can be described by recurrent neural network structures (see also Eqs. (2.1) and (2.2)).

Obviously, if (conventional) propagation techniques were used, the system of differential equations is computed iteratively by use of a time constant (usually 1). This will lead to an insufficient accuracy, especially if real-time synchronisation is required as presented in the application example in Section 3. Only systems with discrete states (e.g. finite state automata [20]) can be computed appropriately. To improve the 'quality' of the propagation process, a smaller time constant $\mathrm{d} t$ or modified propagation methods can be used. A discussion of numerical methods for the computation of (recurrent) neural networks can be found, for example, in [21]. A propagation method for the use in real-time environments was presented in [25]. This approach was motivated by the propagation in Hopfield networks [16] and was developed for the specific application presented in Section 3. The main idea of this approach is to fix the activation of specific neurons and to propagate the network until an energy minimum for this time step is reached, then the propagation for the next time step is started.

\subsubsection{Using modular networks}

Both methods presented above can be used to build a more complex model by modular neural components. For example, a physical system of interacting components can be modelled by designing individual networks for each component, which can be trained separately. This can simplify the design and training process to a great part. In many cases, even the connected system can still be (post-)trained, even if no data of the 'connecting points' is available. An example of a prestructured modular network is presented in Section 3. 


\subsection{Initialisation of prestructured networks by use of a fuzzy system}

As mentioned before it is sometimes useful or even necessary to be able to initialise the parameters (weights) of a neural network appropriately instead of defining them randomly before training. For example, if there is not sufficient training data available but prior expert knowledge in form of linguistic rules can be provided, then an initial (approximate) solution might be given by a problem-specific definition of the network weights. In this case, neuro-fuzzy methods might be more appropriate than pure neural-network-based methods. By now, a number of neuro-fuzzy methods have been developed, which try to combine the interpretability of fuzzy systems with the learning capabilities of feed-forward neural networks $[19,24]$. The main goal of most approaches is to define a model that can be initialised by use of prior expert knowledge (defined by linguistic rules), that can learn from data and that is interpretable before, during and after optimisation and thus, enables a user to extract problem-specific knowledge. Therefore these methods are especially suited for applications were user interaction is desired or necessary.

However, there are still quite a few methods, which try to combine fuzzy systems with recurrent neural networks. For the specific purpose considered in this article a cooperative combination is proposed. That is, both systems may be used independently, but one system modifies the parameters of the other. Here the fuzzy system is used to define the initial parameters of the neural network. Since the use of a prestructured network is proposed, that is designed according to a mathematical model, in principle any method, that can be used to derive the mathematical model parameters, can also be used to initialise its weights. Fuzzy systems [18] have the advantage that they enable the use of linguistic terms to define specific characteristics of a physical system. Thus, they can be easily defined for different domains and by it support even untrained users in defining appropriate initial parameters for the network.

\subsubsection{Fuzzy systems}

The basic idea of fuzzy systems [18] is to define a function based on vague support points in data space. These support points are defined by fuzzy sets which circumscribe the considered region in the input data space. For this, one fuzzy set has to be defined in each considered (input) dimension. Usually a linguistic term like large, small, negative or positive is assigned to each fuzzy set to simplify the definition of the fuzzy rules. The output value assigned to each of these vague support points can be defined by either a fuzzy set (Mamdani-type fuzzy system) or a linear combination of the input values (Sugeno type fuzzy system). In this way for each support point a fuzzy rule is constructed. If the function has to be defined for the complete input data space, the fuzzy sets defining vague support points must overlap and the input data space must be completely covered. A more formal definition of the commonly used Mamdani-type fuzzy system is given in the following.

A Mamdani-type fuzzy system consists of $r$ parallel rules. Let $x_{1}, \ldots, x_{n}$ be $n$ input values, $y_{1}, \ldots, y_{m}$ the $m$ output values and $\mu_{r}^{(1)}, \ldots, \mu_{r}^{(n)}$ and $v_{r}^{(1)}, \ldots, v_{r}^{(m)}$ fuzzy sets of the rule $r$, then the fuzzy rules of a fuzzy system are defined as

$$
\text { if } x_{1} \text { is } \mu_{r}^{(1)} \text { and } \ldots \text { and } x_{n} \text { is } \mu_{r}^{(n)} \text { then } y_{1} \text { is } \mathrm{v}_{r}^{(1)} \text { and ... and } y_{m} \text { is } v_{r}^{(m)} \text {. }
$$


Table 1

Some fuzzy rules for the description of tissue

\begin{tabular}{|c|c|c|}
\hline \multirow{2}{*}{$\begin{array}{l}\text { if hardness is soft and elasticity is big } \\
\text { if shiftability is high }\end{array}$} & then & spring constant is low \\
\hline & then & $\begin{array}{l}\text { spring constant is very low and } \\
\text { viscosity is high }\end{array}$ \\
\hline if consistency is chapped & then & $\begin{array}{l}\text { fraction force is low and } \\
\text { spring constant is very high }\end{array}$ \\
\hline asistency is silg & then & $\begin{array}{l}\text { spring constant is slightly high and } \\
\text { viscosity is low }\end{array}$ \\
\hline
\end{tabular}

To calculate the output of this system, the outputs of every rule are computed first. Then, for all output variables all outputs are combined into a single system output (usually, a fuzzy set). A crisp (exact) output value is derived by a defuzzification procedure, e.g. the centre of gravity of the resulting fuzzy set. For the evaluation of a fuzzy system a t-norm and a t-conorm have to be selected. The t-norm is used to evaluate the activation of each rule and thus the membership degree of the considered point $\left(x_{1}, \ldots, x_{n}\right)$ to the specific vague support point, while the t-conorm is used to combine the output of all rules. Commonly used t-norms for fuzzy systems are the minimum (min) and the product ( prod), commonly used t-conorms are the maximum $(\max )$ and the bounded sum [18].

If a fuzzy system should be used for the initialisation of neural networks in principal two combination strategies can be considered: A hybrid model, where the weights of the network are fuzzified and the neural network is represented by a fuzzy system (e.g. fuzzy perceptron [23]) or a cooperative model, where the fuzzy system is used to compute the weights by using of vague knowledge of the system. For the application considered here, the latter approach is more appropriate, since the recurrent network structure should be preserved to ensure its (mathematical) interpretability and to be able to use optimised propagation procedures. So, to construct a fuzzy system for this specific purpose, the fuzzy rules as well as the membership functions describing the fuzzy sets have to be defined manually by inquiry of experts. If, for example, a fuzzy system for the definition of viscoelastic models by medical experts (as presented in the following section) should be defined, experienced surgeons may be inquired for common terms describing the tissue in their point of view. Based on these terms a fuzzy system that describes the relations between the vague expert knowledge of the object (e.g. very hard, soft, elastic) and the network parameters can be defined. A sample rule base is depicted in Table 1.

\section{A neuro-fuzzy method to define and simulate viscoelastic models}

Viscoleastic models were introduced by Terzopolous in 1988 [40] to simulate object deformations in virtual reality. The main goal was to obtain a visual convincing 
simulation at an acceptable interactive frame-rate; a most detailed and realistic physical model was less important. Graphic representations of static geometry build with commonly used modelling tools (e.g. VRML-Editors) can be directly used for the creation of the outer shape of viscoelastic models [31]. Another reason to choose viscoelastic models is that the mesh structure can be easily transferred into a network structure. Furthermore, it is easy to perform cuts in a mesh-based model.

As in other physical-based approaches, the main problem is to find the necessary physical parameters for the model. Wrong parameter definition leads in most cases to an unrealistic simulation. Using the described neuro-fuzzy techniques to define and simulate viscoelastic models, the physical parameters can be learned from real-life measurement of deformed bodies or initialised by fuzzy rules.

\subsection{Viscoelastic models}

Viscoelastic models are an extension of spring-mass models [12], which were initially described by linear spring laws without viscosity. Viscoelastic models consist of simple viscoelastic elements that define a physical model of two mass points which are connected by a spring-damper element (Fig. 2a). In viscoelastic models the whole mass of the object is divided up between the mass points of the model, which are represented as nodes in a mesh (see the simple mesh in Fig. 2a). Thus, every node of the mesh can be connected elastically and/or viscously to its neighbours.

The occurring forces in such models are be defined by the following physical laws (for details see, e.g. $[12,40]$ ). The forces at the nodes (mass points) are defined by

$$
\sum_{i} F_{i}=F=m a
$$

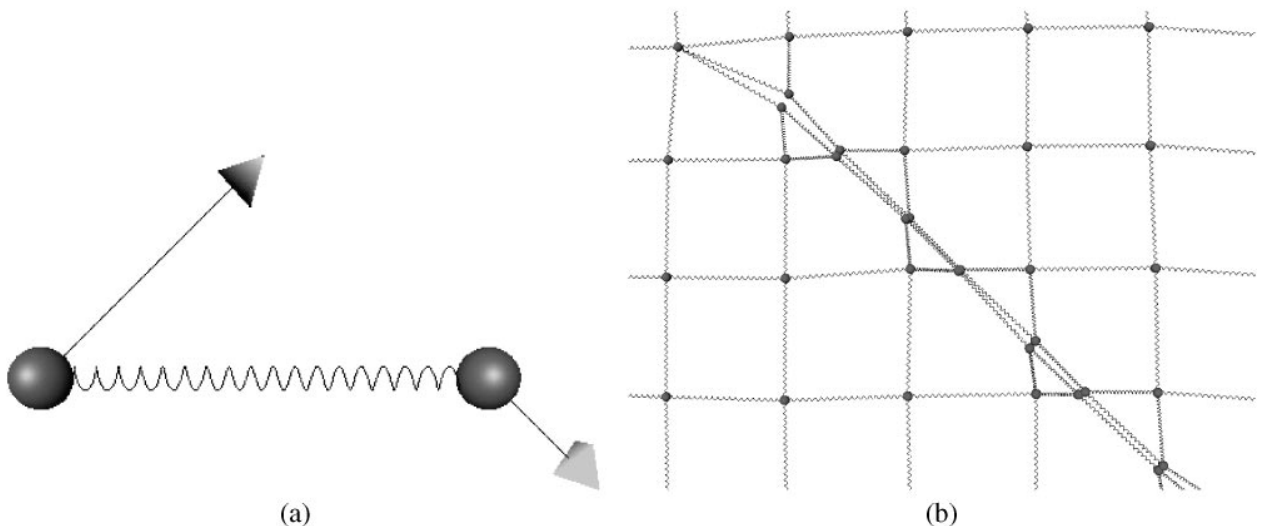

(a)

(b)

Fig. 2. Viscoelastic element (a) and a simple viscoelastic surface model with a diagonal cut (b). 
where $m$ defines the mass of the node, $a$ the acceleration of the node and $F_{i}$ the forces of the connected viscoelastic elements. The resulting forces at the viscoelastic elements are defined by

$$
F_{i}=F_{s}(l, v)-F_{d}(l, v)
$$

where $F_{s}(l, v)$ defines the spring force, $F_{d}(l, v)$ defines the damping force or viscosity, $l$ defines the length and $v$ the relative velocity of the connected mass points. In case of linear spring models the occurring forces on these elements are defined as

$$
F_{i}=c\left(l_{\text {norm }}-l\right)-d v
$$

where $c$ is the spring constant, $d$ is the viscosity or damping constant, $l_{\text {norm }}$ is the length of the element when no external force is applied.

One advantage of this approach is the possibility to simulate transections by disconnecting elements. The elements along the transection line are split up and new nodes are connected to each of the loose endings (see Fig. 2b). Furthermore, to every node of the mesh, external forces can be applied (for example gravity or collision forces). The internal forces and the displacement of the nodes are computed correspondingly. Also, if the displacement of a node is given, the resulting (inner) forces can be calculated. By use of this model structure inertia and torque (e.g. in case of object rotation) can be simulated as well [29].

\subsection{The network model}

As presented in Section 2.3, a network model can be defined based on the mathematical description of the physical model. To simplify the creation of the usually complex objects, two neuron groups (modules or building blocks) are defined that are used to build the model by modular blocks of local interaction. The structure of the modules implements the system of differential equations, which defines the viscoelastic model. Two different network modules are used for this purpose: the mass nodes and the viscoelastic nodes. In the following, a brief description of the model structure is given. A more formal definition can be found in [25].

\subsubsection{The mass node's dynamic}

The neurons determining the mass dynamics (inertia) are defined by three neurons, which calculate the position $\vec{p}$, velocity $\vec{v}$, and acceleration $\vec{a}$ of the mass point (see Fig. 3). Internal (forces from connected springs) or external forces $\vec{F}_{i}$ can be applied to the acceleration neuron, which calculates the actual acceleration using the node's mass $m$. The velocity and position neurons are self-connected feedback nodes. These neurons are used as integrators to calculate the velocity and position (see Section 2.3). 


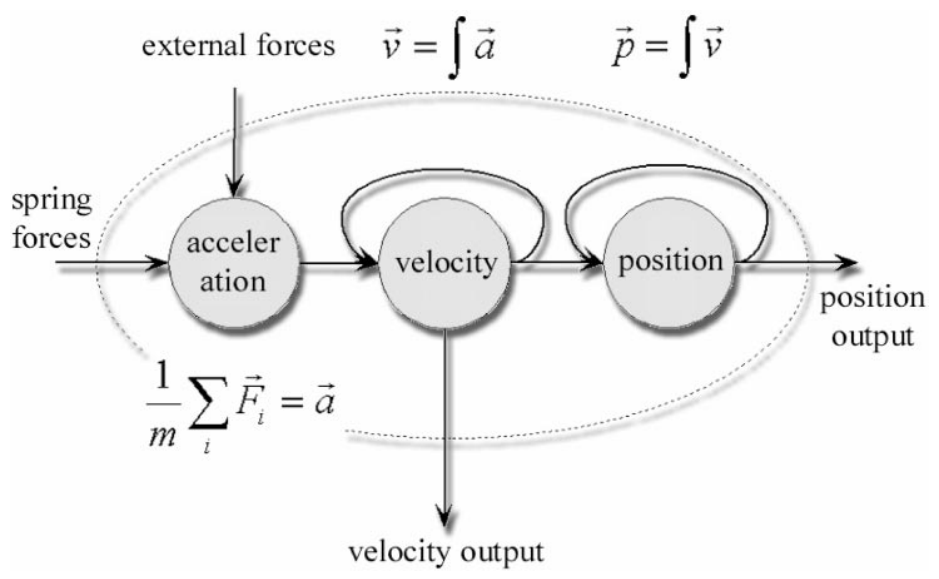

Fig. 3. Neurons describing the mass point dynamic.

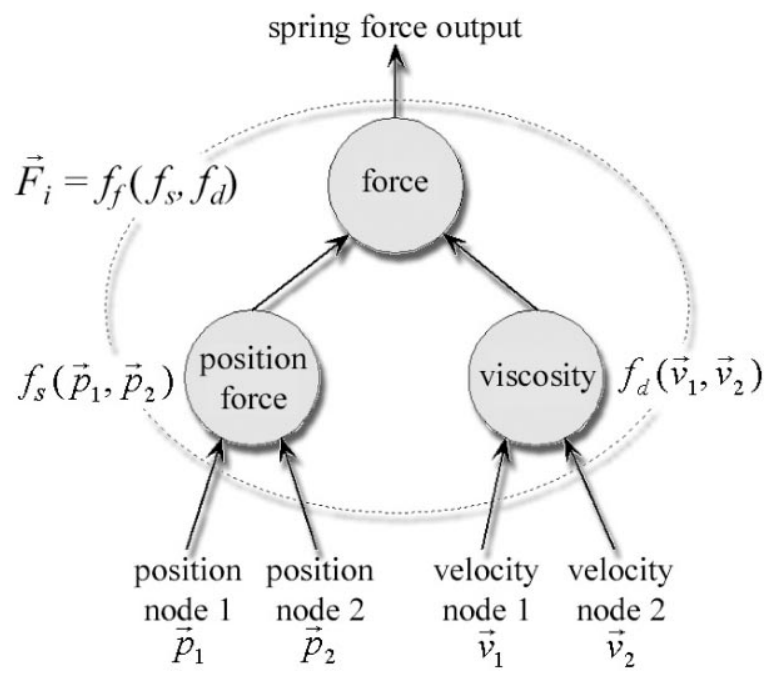

Fig. 4. Neurons describing the dynamic of the viscoelastic element.

\subsubsection{The viscoelastic node's dynamic}

The modules determining the viscoelastic dynamic (see Fig. 4) calculate the actual total spring force $\vec{F}$, based on the positions $\left(\vec{p}_{1}, \vec{p}_{2}\right)$ and velocities $\left(\vec{v}_{1}, \vec{v}_{2}\right)$ of the connected nodes. The position force neuron calculates a force function $f_{s}\left(\vec{p}_{1}, \vec{p}_{2}\right)$. If - for comparison with traditional methods - linear models should be used, the force function can be defined as:

$$
f_{s}\left(\vec{p}_{1}, \vec{p}_{2}\right)=\left(\vec{p}_{1}-\vec{p}_{2}\right)\left(\frac{l_{\text {norm }}}{\left|\vec{p}_{1}-\vec{p}_{2}\right|}-1\right),
$$


where $l_{\text {norm }}$ is the initial or undeformed length of the viscoelastic element. (The transfer function (3.19) can be implemented by networks with multiplicative connections [28]. These functions are not needed for the derivation of the learning algorithm presented in the following, so they are omitted for simplification.) The viscosity neuron calculates the force function $f_{d}\left(\vec{v}_{1}, \vec{v}_{2}\right)$ (e.g. for linear models: $\left.f_{d}\left(\vec{v}_{1}, \vec{v}_{2}\right)=\vec{v}_{1}-\vec{v}_{2}\right)$.

The force neuron calculates the actual spring force, which depends on the results of the functions $f_{s}$ and $f_{d}$. In case of linear models this could be, for example, $\vec{F}_{i}=c f_{s}-d f_{d}$, where $c$ is the spring constant and $d$ is the viscosity or damping constant. Thus, by use of the functions $f_{s}, f_{d}$ and $f_{f}$ any linear or non-linear viscoelastic dynamic can be implemented. Therefore, it resolves the insufficient biomechanical realism of linear viscoelastic models [8].

\subsection{Object modelling}

One main difficulty developing virtual environments is to create appropriate object models. The easiest way to create these models is to use a 3D CAD- or modelling tool (e.g. VRML-modeler, Alias|Wavefront Maya). Nearly all 3D models that are created by modelling software, consist of sets of connected triangles or triangular meshes. These meshes can be converted to viscoelastic models by replacing the edges of the triangles with viscoelastic elements and the vertices with nodes. To simplify the design, the program Elastodynamic Shape Modeler has been developed [43], which transforms triangular meshes into viscoelastic models (Fig. 5). The geometric description of the objects can be imported directly from the used modelling tool. A fuzzy rule base, as described in Section 2.4, initialise the parameters of the neural network.

\subsection{Learning}

The presented learning algorithm uses the positions of specific object points during discrete time steps to learn or optimise the required (physical) parameters. The time-series data for learning can be derived, for example, by a time-dependent optical measurement of an object under influence of external forces.

The goal of the learning method is to derive the parameters of the physical viscoelastic model, the masses $m_{j}$ and the constants $c_{i}$ and $d_{i}$. Therefore, the learning algorithm just modifies the weights defined by these constants during learning.

To simplify the description of the learning algorithm, a simple network module is used, consisting of two nodes that are connected by a viscoelastic element (Fig. 6).

The group of mass-node-neurons consists of three neurons (see also Section 3.2): The position-neuron calculates the position $p$ of the assigned node based on its current 


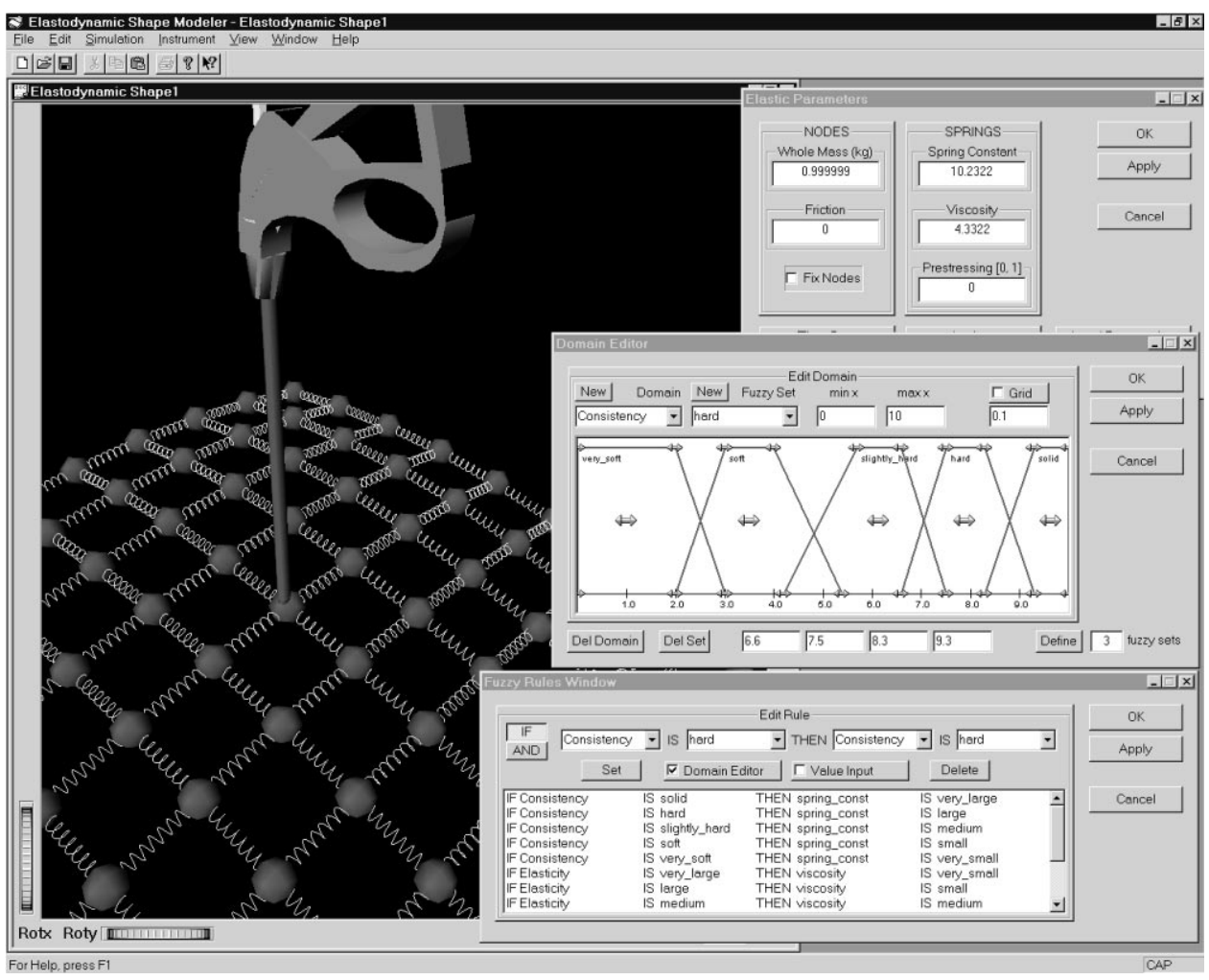

Fig. 5. Screenshot of the tool elastodynamic shape modeler.

position and its velocity, the velocity-neuron calculates the velocity $v$ based on the nodes velocity and its acceleration and the acceleration-neuron, which calculates the nodes acceleration $a$ based on the forces of the connected springs and external forces. With $t_{c}$ be the time constant for the simulation, these neurons are defined as

$$
\begin{aligned}
& \vec{o}_{p}(t)=\vec{f}_{p}\left(n \vec{e} t_{p}(t)\right)=t_{c} \vec{o}_{v}(t)+\vec{o}_{p}(t-1) \\
& \vec{o}_{v}(t)=\vec{f}_{v}\left(n \vec{e} t_{v}(t)\right)=t_{c} \vec{o}_{a}(t)+\vec{o}_{v}(t-1) \\
& \vec{o}_{a}(t)=\vec{f}_{a}\left(n \vec{e} t_{\mathrm{a}}(t)\right)=w_{m}\left(\sum_{i} w_{i} \vec{o}_{f_{1}}(t)+e \overrightarrow{e x} t_{f}(t)\right)
\end{aligned}
$$

where $o_{\mathrm{f}_{i}}$ is the force of the connected spring $i . w_{i}$ defines the direction of the force (see Fig. 6), $w_{m}$ : $=m^{-1}$ and $m$ is the mass of the node, and $e x t_{f}$ is an external force applied to the node. 


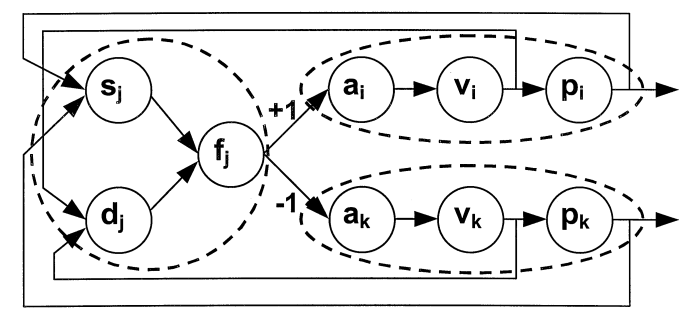

Fig. 6. Two nodes $(i$ and $k$ ) connected by a viscoelastic element $(j)$.

The group of viscoelastic-neurons consists of three neurons: The position-forceneuron calculates the static spring force $f_{s}$ based on the positions of the connected nodes, the viscosity-neuron calculates the damping force $f_{d}$ based on the relative velocities of the connected nodes and the force-neuron, which combines these forces to a single output force $f_{f}$ of the viscoelastic element. These neurons are defined as

$$
\begin{aligned}
& \vec{o}_{f}(t)=\vec{f}_{f}\left(n \vec{e} t_{f}(t)\right)=c_{f} \vec{o}_{s}(t)-d_{f} \vec{o}_{\mathrm{d}}(t), \\
& \vec{o}_{s}(t)=\vec{f}_{s}\left(n \vec{e} t_{s}(t)\right)=\vec{f}_{s}\left(\vec{o}_{p_{1}}(t)-\vec{o}_{p_{2}}(t)\right), \\
& \vec{o}_{d}(t)=\vec{f}_{d}\left(n \vec{e} t_{\mathrm{d}}(t)\right)=\vec{f}_{d}\left(\vec{o}_{v_{1}}(t)-\vec{o}_{v_{2}}(t)\right),
\end{aligned}
$$

where $c_{f}$ defines the physical spring constant, $d_{f}$ the viscosity constant, and $f_{s}, f_{d}$ the spring and viscosity functions of the viscoelastic element. Connected groups of two node-neurons and one spring-neuron are depicted in Fig. 6.

The presented learning algorithm performs a single backpropagation step in time after propagation of each time step, and modifies the weights - different to the BPTT algorithm - immediately. To prevent a large deviation from the required positions a 'teacher forcing' strategy is used: After a fixed number of time steps, the outputs $o_{p}(t)$ (respectively the activations) of the position-neurons are set to the required values $p(t)$.

The learning algorithm uses a similar error function as defined by Eqs. (2.4) and (2.5), except that only the position-neurons are considered for the calculation of the error, since the velocity, acceleration or spring forces are usually not available for training. Therefore, the total error $E$ is defined as

$$
E=\sum_{t=0}^{T} E(t)=\sum_{t=0}^{T}\left[\frac{1}{2} \sum_{p}\left(\vec{E}_{p}(t)\right)^{2}\right]
$$

with

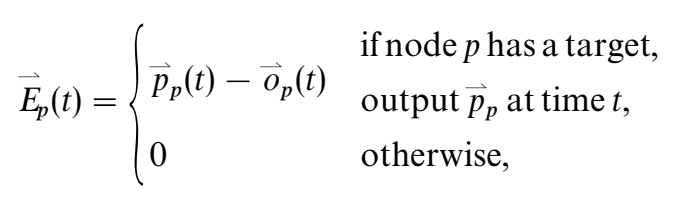


where $o_{p}(t)$ is the position of node $k$ and $p_{k}(t)$ the position of the node $k$ in the exact physical model at time-step $t$. According to the gradient-descent method, the error rates for the respective neurons are derived as follows (see also Eqs. (2.7) and (2.9)).

Let $p(t)$ be the desired output at time $t$ for an outer position-neuron, for which an target output exists, then the error rate $\delta_{p}(t)$ is defined as:

$$
\vec{\delta}_{p}(t)=-\frac{\partial \vec{E}(t)}{\partial n \vec{e} t_{p}(t)}=\vec{p}(t)-\vec{o}_{p}(t) .
$$

For the error rates of a velocity-neuron and an acceleration-neuron this leads to

$$
\begin{aligned}
& \vec{\delta}_{v}(t)=-\frac{\partial \vec{E}(t)}{\partial n \vec{e} t_{\mathrm{v}}(t)}=\vec{\delta}_{p}(t) t_{c}=\left(\vec{p}(t)-\vec{o}_{p}(t)\right) t_{c}, \\
& \vec{\delta}_{a}(t)=-\frac{\partial \vec{E}(t)}{\partial n \vec{e} t_{a}(t)}=\vec{\delta}_{v}(t) t_{c}=\left(\vec{p}(t)-\vec{o}_{p}(t)\right) t_{c}^{2} .
\end{aligned}
$$

With Eq. (3.30) the weight-gradient for $w_{m}$ can be defined as

$$
\Delta w_{m}(t)=\eta \delta_{a}(t) \vec{o}_{s}(t)=\eta\left(\vec{p}(t)-\vec{o}_{p}(t)\right) t_{c}^{2} \vec{o}_{s}(t),
$$

where $\eta$ is a learning rate. Since $t_{c}$ is constant, we define

$$
\Delta w_{m}^{*}(t)=\eta_{m}\left(\vec{p}(t)-\vec{o}_{p}(t)\right) \vec{o}_{f}(t)
$$

with $\eta_{m}$ be a learning rate. The error rates for the adaption of the spring constant can be obtained in the same way:

$$
\vec{\delta}_{f}(t)=-\frac{\partial \vec{E}(t)}{\partial \overrightarrow{n e} t_{f}(t)}=-\frac{\partial \vec{E}(t)}{\partial \vec{o}_{f}(t)} \frac{\partial \vec{o}_{f}(t)}{\partial n \vec{e} t_{f}(t)}
$$

With Eq. (3.22) this leads to

$$
\frac{\partial \vec{E}(t)}{\partial \vec{o}_{f}(t)}=\sum_{a} \frac{\partial \vec{E}(t)}{\partial n \vec{e} t_{a}(t)} \frac{\partial n \vec{e} t_{a}(t)}{\partial \vec{o}_{f}(t)}=-\sum_{a} w_{a} \vec{\delta}_{a}(t) .
$$

So, $\delta_{f}(t)$ is defined as

$$
\vec{\delta}_{f}(t)=\sum_{a} w_{a} \vec{\delta}_{a}(t)
$$


With Eq. (3.35) the weight-gradients for the spring constants $c_{f}$ and $d_{f}$ can be defined

$$
\begin{aligned}
& \Delta w_{c}(t)=\eta \vec{\delta}_{f}(t) \vec{o}_{s}(t), \\
& \Delta w_{d}(t)=-\eta \vec{\delta}_{f}(t) \vec{o}_{d}(t),
\end{aligned}
$$

where $\eta$ is a learning rate. The error rates for the position-force-neurons are defined as

$$
\vec{\delta}_{s}(t)=f_{s}^{\prime}\left(n e t_{s}(t)\right) \vec{\delta}_{f}(t) w_{1}=f_{s}^{\prime}\left(n e t_{s}(t)\right) \sum_{a} \vec{\delta}_{a}(t) .
$$

The error rates for the viscosity-neurons are obtained in the same way, therefore:

$$
\vec{\delta}_{d}(t)=-f_{d}^{\prime}\left(n e t_{d}(t)\right) \sum_{a} \vec{\delta}_{a}(t)
$$

Finally, the error rates $\vec{\delta}_{p}^{*}(t)$ for inner position-neurons, for which no target output $p(t)$ for learning is available, have to be derived

$$
\vec{\delta}_{p}^{*}(t)=-\frac{\partial \vec{E}(t)}{\partial n \vec{e} t_{p}(t)}=-\frac{\partial \vec{E}(t)}{\partial \vec{o}_{p}(t)} \frac{\partial \vec{o} p(t)}{\partial n \vec{e} t_{p}(t)}
$$

with

$$
\frac{\partial \vec{E}(t)}{\partial \vec{o}_{p}(t)}=\sum_{i \in\{s, d\}} \frac{\partial \vec{E}(t)}{\partial n \vec{e} t_{i}(t)} \frac{\partial n \vec{e} t_{i}(t)}{\partial \vec{o}_{p}(t)}=-\sum_{s} \vec{\delta}_{s}(t)-\sum_{d} \vec{\delta}_{d}(t) .
$$

$\vec{\delta} \underset{p}{*}(t)$ for an inner position-neurons is defined as

$$
\vec{\delta} \underset{p}{*}(t)=\sum_{s} \vec{\delta}_{s}(t)+\sum_{d} \vec{\delta}_{d}(t) .
$$

Therefore, if no time series data $p(t)$ is available for an inner position-neuron, then the error is propagated further back by use of Eq. (3.42) and $\vec{\delta}_{p}(t)$ in Eq. (3.29) is replaced by $\vec{\delta} *(t)$.

The learning algorithm adapts the weights by use of Eqs. (3.31), (3.36) and (3.37) for every node. A pseudocode description of the algorithm is given in Fig. 7.

If the object to be simulated consists of homogenous material and the network structure was suitably defined, all modules can share the same weights. So the constants $(c, m$ and $s$ ) can be learned jointly as coupled weights. In this case, the training process is comparable to an epoch learning of a single network module. Thus, the performance and the stability of the learning algorithm can be improved. Even areas of the same material can be jointly processed, by slight modifications of the algorithm. Furthermore, in many cases, the mass $m_{j}$ of the nodes can be 


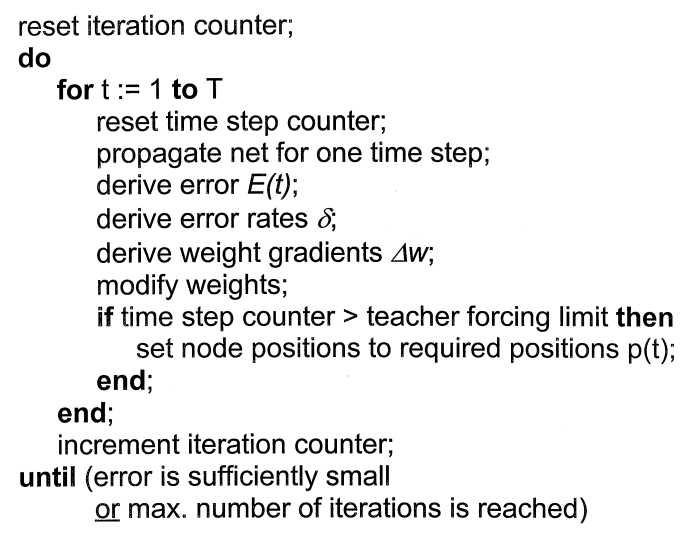

Fig. 7. Learning algorithm for a viscoelastic model network.

predefined, by just dividing up the total mass of the object between the mass nodes of the network.

\subsection{Learning example}

To demonstrate the usability of the presented algorithm a very simple but typical measurement problem was used: An object is deformed over a period of time by a constant external force [26]. As object a simple cube with diagonal springs, consisting of 27 nodes and 86 springs (see Fig. 8) with predefined 'physical' parameters for the masses and springs was simulated. The bottom nodes of the cube were fixed to the ground during simulation. To the external node in the centre of the top layer a constant force was applied. The deformation of the object was simulated for a period of 250 time steps. After this period, the object's nodes rest in a final position - an attractor or energy minimum. The positions of the nodes on the discrete time steps were stored as learning data.

After this generation of test data the learning process was started. For the first sample the mass, spring and viscosity constants were randomly chosen in an interval defined by $[0.2 x, 5 x]$ around the original settings $x$. The algorithms was initialised with a learning rate $\eta=0.01$ for the spring constants $c_{i}$ and $s_{i}$, and $\eta_{m}=0.1$ for the mass constants $m_{j}$. A teacher forcing limit of ten iteration steps was chosen. The algorithm was terminated after 100 learning cycles (each cycle is a complete propagation through time) with an error $E=1.21$. The error after each training cycle is depicted in Fig. 9. As expected, due to the low amount of (different) training data, the algorithm did not retrieve the 'physical' parameters of the model. The resulting constants defining $c_{i}, s_{i}$ and $m_{j}$ were still spread around their 'exact' settings. This can lead to an undesired behaviour of the object in untrained situations.

The second example was initialised as above, but with a learning rate of $\eta=0.1$ for the spring constants. Furthermore, the masses were predefined and not modified 


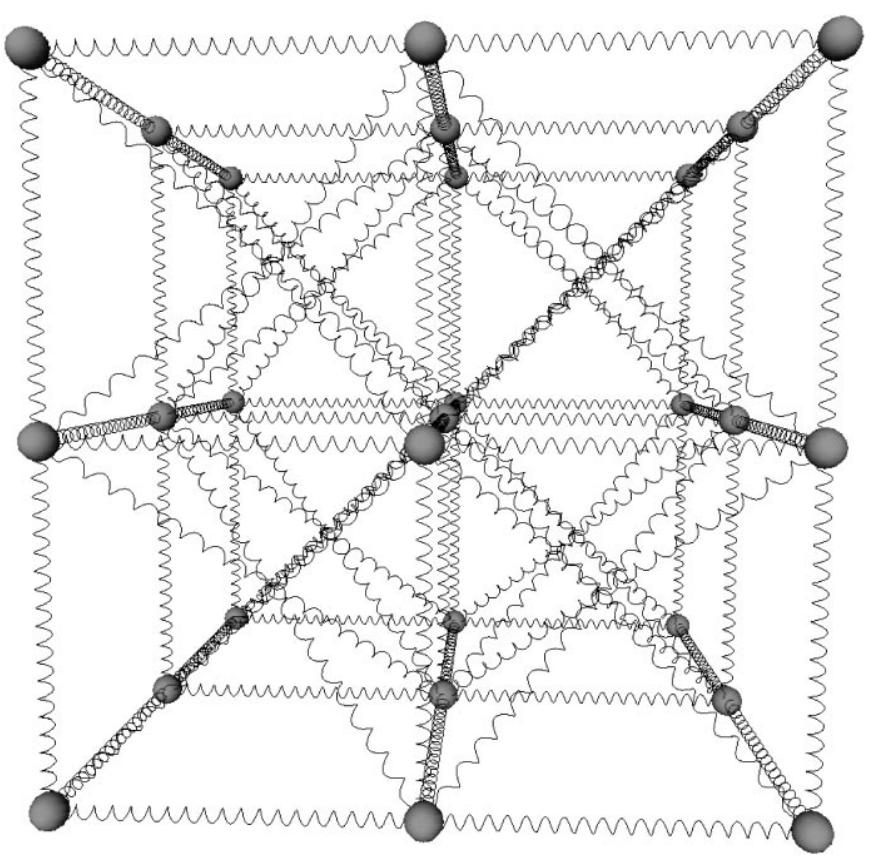

Fig. 8. Elastic cube used for learning.

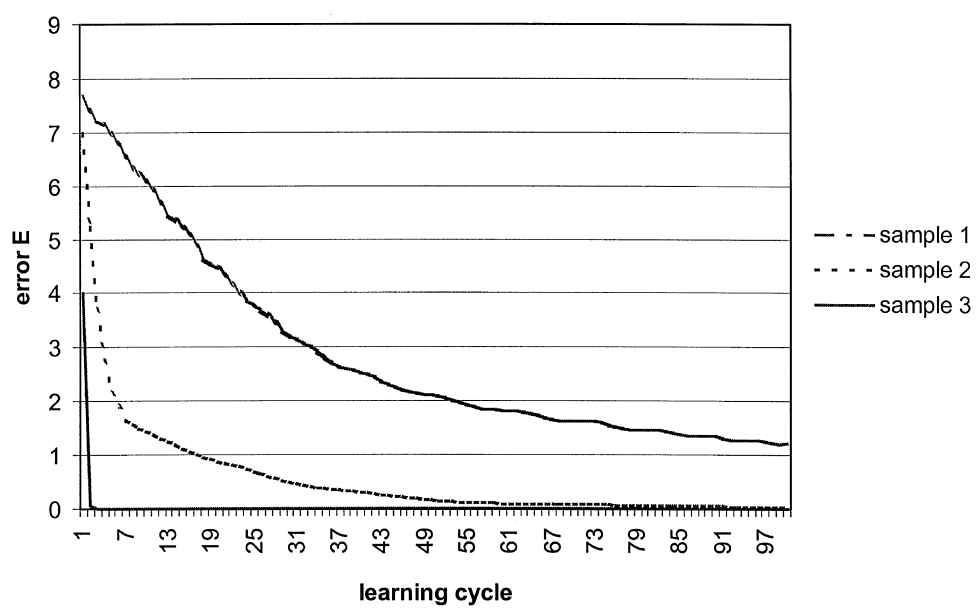

Fig. 9. Learning example: error $E$.

during learning. The algorithm was again terminated after 100 learning cycles, with an achieved error $E=0.033$. The error after each training cycle is depicted in Fig. 9. Again, the algorithm did not retrieve the 'physical' parameters of the model. The resulting constants were still spread around their 'exact' settings. 
In the last example the spring constants $c$ and $s$ were learned jointly for all springs. The algorithm was initialised as above, but the masses were predefined and an offset was added to the spring and mass constants to prevent an accidental parameter match. The algorithm was again terminated after 100 learning cycles. The algorithm achieved an error of $E=0.138$. This is slightly higher than in the other examples. The error after each training cycle is depicted in Fig. 9. In this case the algorithm was able to retrieve the physical parameters of the model, with an error of about less than $0.1 \%$.

\section{Application example: Virtual surgery}

Nowadays, computer-assisted surgery is already used for procedure training and operation planning but most of the utilised methods are based on static visualisation techniques. To improve the benefit for surgical training, a visual convincing static modelling of the operation scenario and the involved tissues is not sufficient, because tissues can be deformed at contact, and transections can be made. Especially for the interaction with medical devices, such as endoscopic instruments, it is necessary to simulate the deformation of tissue under influence of collision forces $[8,9,38]$.

Currently, there are a number of simulators under development, which allow physical-based, real-time deformations of virtual tissues and organs (see, for example $[5,39]$ ). Most of these simulators use FEM for computing virtual deformations because FEM are well known to solve similar problems in non real-time environments. However, to meet the real-time requirement these methods have to be simplified and therefore loosing their realism. Another problem is that the required parameters for the models are difficult to obtain because living tissues have inhomogeneous deformation behaviour. Additionally, convincible methods to measure the physical parameters for a deformation model of these tissues are not developed so far.

For these reasons the surgical simulation was chosen as a first application to test our neuro-fuzzy model. Although the simulation of deformable tissues can be used in a wide field of surgical training (e.g. $[4,8,9,38]$ ), the main field of application is the minimally invasive surgery because realistic interface devices for the simulation of endoscopic instruments already exists [31], minimally invasive interventions require much more training as conventional surgery, and the increasing number of minimally invasive interventions.

The first surgical simulator, in which the presented neuro-fuzzy system was used to simulate deformable objects, was the simulation system SUSILAP-G (SUrgical SImulator for LAParoscopy in Gynaecology) (see Fig. 10). Because many surgical interventions in gynaecology aim at operations of the fallopian tube, a sterilisation was chosen for a virtual intervention. The deformation behaviour of the fallopian tube was described by fuzzy rules, which were optimised in collaboration with a professional surgeon [30]. Because of the resulting realism no further optimisation with the neural network was necessary. 


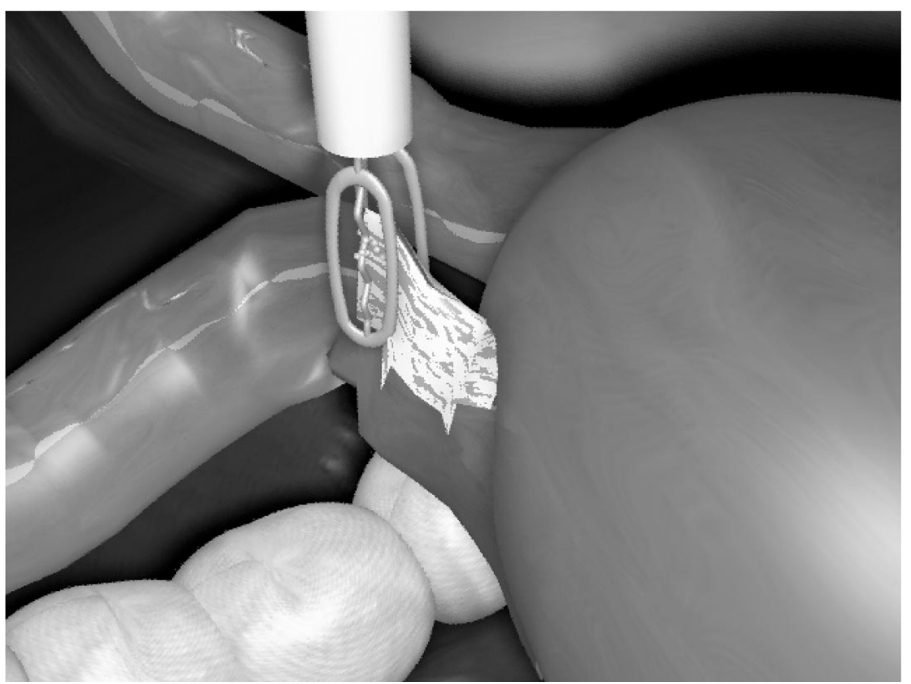

Fig. 10. Virtual deformation of the fallopian tube with SUSILAP-G.

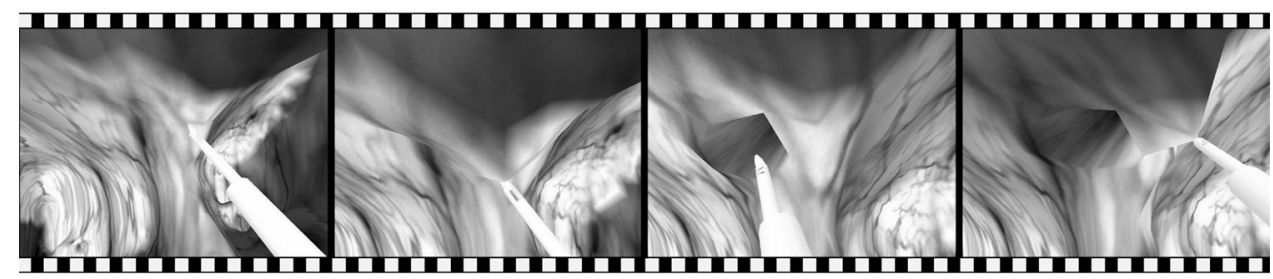

Fig. 11. Virtual local tissue deformation and fragmentation inside the ventricle. From left to right: Unmanipulated ventricle, virtual deformation by using a biopsy forceps, virtual fragmentation simulated by moving some of the nodes normal to the surface gradient and fixing them, virtual deformation near the old fragmentation.

Another system using neuro-fuzzy deformation is a simulator for minimally invasive neurosurgery, called ROBO-SIM [17] (see Fig. 11), which is developed as part of ROBOSCOPE (project of the European Community's telematics program). Minimally invasive neurosurgery is mainly of importance for operation of diseases in the central area of the brain, which are accessible for the surgeon only by transgression of healthy brain tissue. Besides occlusive hydrocephalus and cystic brain tumours, ventricular tumours, i.e. tumours within the system of liquid filled cavities in the brain (ventricles), are the major indications for minimally invasive neurosurgery. The main goal of the latter technique is to keep the surgical access through viable brain tissue as small as possible, which requires many years of training. Because deformations occur inside the liquid filled cavities, the deformation's viscosity varies locally, and there is also friction between the tissue surface and the liquid. Therefore, a visual convincing 
deformation is difficult to obtain and a first initialisation with a fuzzy system did not lead to a sufficient result [2]. Therefore, a very promising possibility developed as part of the project ROBOSCOPE can be used to measure the deformation of the brain tissue: the coregistration of real-time 3D ultrasound and pre-operative MR data sets [34]. In ROBOSCOPE this is used to match the cheep and low-quality 3D ultrasound data sets with the high-quality, very expensive and time-consuming MR data sets, which are created before the operation. Then, the MR data set is recalculated to match the current state of the brain. This is necessary due to the brain shift resulting from pressure changing caused by opening the skull. The brain shift is a deformation of up to a few centimetres of the brain. To match the different data sets a field of deformation vectors are calculated, which can be directly used to train the neural network.

\section{Conclusions}

As presented, physical systems can be modelled or identified by neuro-fuzzy techniques. By use of learning methods, measured data of the system can be used to derive or optimise the parameters of the obtained model. It is possible to initialise the learning process by prior knowledge based on 'real' physical parameters or parameters defined by a fuzzy system.

The presented specific neuro-fuzzy model for viscoelastic object modelling and simulation was designed in particular to support a developer of virtual environments. By use of the presented learning algorithm, measured data (e.g. derived from an optical measurement of a deformable solid) can be used to derive the parameters of viscoelastic models. In most cases this should lead to a sufficient realistic simulation even if the structure of the viscoelastic model is unfavourably defined. Nevertheless, further research is still necessary.

The learning process can be initialised by use of prior knowledge based on 'real' physical parameters or parameters defined by the tool Elastodynamic Shape Modeler [31]. Since the existing propagation algorithms for these types of networks can be used in real-time applications, the resulting network is able to simulate objects in virtual environments.

It should be noted that the 'physical' parameters, achieved by use of the presented learning algorithm, need not match the parameters of the physical model, if there is not enough training data with different deformations available. In this case the algorithm may derive a combination of parameters, which follows the same (trained) trajectory, but result in different inner forces. Nevertheless, since the main objective of this algorithm is to find a combination of parameters resulting in a visual convincing simulation of the object, 'exact' modelling is not necessary. To improve the learning performance, known homogenous areas should share the same parameters and if the masses of the nodes are known, they should be used to set the mass constants fixed. Thus, the algorithm is able to derive a more 'exact' model. 
The simulation model is already integrated in different simulators for minimallyinvasive surgery in gynaecology [30], hand surgery [41] and neurosurgery $[17,32]$. Since the deformation behaviour of tissues is inhomogeneous, the parameter adaptation of the simulating model is very important. Though, in some cases an initialisation by the fuzzy-system is appropriate to obtain a sufficient impression of reality. Complex virtual anatomical structures will only behave like real ones if the physical parameters are automatically adapted. First approximations of the parameters can be obtained using surface deformation data of organs, which are already removed from the body. However, these organs have different deformation behaviours than living tissue, so training with these models will be almost the same as the traditional training with post-mortems. Therefore, a very promising task will be the deformation measurement of living tissues by use of 3D ultrasound. Based on these data sets an application-oriented verification of the presented model by use of complex anatomical structures will be possible then.

\section{References}

[1] J.E. Akin, Application and Implementation of Finite Element Methods, Academic Press, London, 1982.

[2] L.M. Auer, A. Radetzky, C. Wimmer et al., Visualization for planning and simulation of minimally invasive neurosurgical procedures, Proceedings of MICCAI, Lecture Notes in Computer Science, Springer, Berlin, 1999, pp. 1199-1209.

[3] J. Avis, N.M. Briggs, F. Kleinermann et al., Anatomical and physiological models for surgical simulation, Proceedings of Medicine Meets Virtual Reality 7, IOS Press Amsterdam, 1999, pp. 23-29.

[4] C. Basdogan, P. Loan, J.M. Rosen et al., An interactive model of the human thigh for simulation surgical procedures in virtual environments, Proceedings of the Winter Annual Meeting of ASME, 1996.

[5] J. Berkley, S. Weghorst, H. Gladstone et al., Fast finite element modeling for surgical simulation, Proceedings of Medicine Meets Virtual Reality 7, IOS Press, Amsterdam, 1999, pp. 55-61.

[6] P.G. Ciarlet, J.L. Lions (Eds.), Handbook of Numerical Analysis, Vol. III, North-Holland, Amsterdam, 1994.

[7] A. Cichocki, R. Unbehauen, Neural Networks for Optimization and Signal Processing, Wiley, New York, 1994.

[8] S. Cotin, H. Delingette, N. Ayache, Efficient linear elastic models of soft tissues for real-time surgery simulation, INRIA Report No. 3510, INRIA Sophia Antipolis, France, 1998.

[9] S.L. Delp, P. Loan, C. Basdogan et al., Surgical simulation: an emerging technology for training in emergency Medicine, Teleoperators Virtual Environ. 6 (4) (1997) 147-159.

[10] C.S. Desai, J.F. Abel, Introduction to the Finite Element Method, Van Nostrand Reinhold, New York, 1972.

[11] E. Domany, J.L. van Hemmen, K. Schulten (Eds.), Models of Neural Networks II, Springer, New York, 1994.

[12] P.L. Gould, Introduction to Linear Elasticity, Springer, New York, 1994.

[13] C.W. Groetsch, Inverse Problems in the Mathematical Sciences, Vieweg, Braunschweig, 1993.

[14] R. Grzeszczuk, D. Terzopoulos, Geoffrey Hinton, NeuroAnimator: fast neural network emulation and control of physics-based models, SIGGRAPH 98 Conference Proceeding, 1998. 
[15] S. Hayken, Neural Networks, Prentice-Hall, Englewood Cliffs, NJ, 1994.

[16] J.J. Hopfield, Neural networks and physical systems with emergent collective computational abilities, Proc. Nat. Acad. Sci. USA, 79 (1982) 2554-2558.

[17] G. Kleinszig, A. Radetzky, C. Wimmer, D.P. Pretschner, L.M. Auer, ROBO-SIM: a simulator for minimally invasive interventions, Scientific Program of the RSNA, 209(P),Chicago, 1998, p. 682 .

[18] R. Kruse, J. Gebhardt, F. Klawonn, Foundations of Fuzzy Systems, Wiley, New York, 1994.

[19] C.T. Lin, C.S.G. Lee, Neural Fuzzy Systems: a Neuro-Fuzzy Synergism to Intelligent Systems, Prentice-Hall, Englewood Cliffs, NJ, 1996.

[20] P. Manolios, R. Fanelli, First-order recurrent neural networks and deterministic finite state automata, Neural Comput. 6 (6) (1994) 1155-1173.

[21] M.V. Mascagni, A.S. Sherman, Numerical methods for neuronal modeling, in: Christof Koch Idan Segev (Eds.), Methods in Neuronal Modeling, MIT Press, Cambridge, MA, 1998.

[22] M.L. Minsky, S.A. Papert, Perceptrons (2nd expanded edition 1988), MIT Press, Cambridge, MA, 1969.

[23] D. Nauck, A fuzzy perceptron as a generic model for neuro-fuzzy approaches, Proceedings of the second German GI-Workshop Fuzzy-Systeme'94, Munich, Germany, 1994.

[24] D. Nauck, F. Klawonn, R. Kruse, Foundations of Neuro-Fuzzy Systems, Wiley, New York, 1997.

[25] A. Nürnberger, A. Radetzky, R. Kruse, A problem specific recurrent neural network for the description and simulation of dynamic spring models, Proceedings of the International Joint Conference on Neural Networks, Anchorage, AK, May 1998, pp. 468-473.

[26] A. Nürnberger, A. Radetzky, R. Kruse, Determination of elastodynamic model parameters using a recurrent neuro-fuzzy system, Proceedings of the seventh European Congress on Intelligent Techniques and Soft Computing (EUFIT'99), Verlag Mainz, Aachen, 1999.

[27] F.J. Pineda, Recurrent backpropagation networks, in: Y. Chauvin, D.E. Rumelhart (Eds.), Backpropagation: Theory, Architectures and Applications, 1995, pp 99-135.

[28] J.B. Pollak, Cascaded backpropagation on dynamical connectionist networks, Proceedings of the Ninth Conference Cognitive Science Society, Seattle, WA, 1987, pp. 391-404.

[29] A. Radetzky, A. Nürnberger, D.P. Pretschner. A neuro-fuzzy approach for the description and simulation of elastic tissues, Multimedia Technology in Medical Training, Proceedings of the European Workshop, 1997, pp 30-40.

[30] A. Radetzky, A. Nürnberger, D.P. Pretschner, Simulation of elastic tissues in virtual medicine using neuro-fuzzy systems, Medical Imaging 1998: Image Display, Proceedings of SPIE, Vol. 3335, 1998, pp. 399-409.

[31] A. Radetzky, A Nürnberger, M. Teistler, D.P. Pretschner, Elastodynamic shape modeling in virtual medicine, International Conference on Shape Modeling and Applications, IEEE Computer Society Press, Piscataway, NJ, 1999, pp. 172-178.

[32] A. Radetzky, Ch. Wimmer, G. Kleinszig, M. Brukner, L.M. Auer, D.P. Pretschner, Interactive deformable volume graphics in surgical simulation, Proceedings of the International Workshop on Volume Graphics, Swansea, GB, (1996) 19-27.

[33] A. Rappoport, A. Sheffer, M. Bercovier, Volume-preserving free-form solids, IEEE Trans. Visualization Comput. Graphics, 2 (1) (1996) 19-27.

[34] A. Roche, G. Malandain, N. Ayache, S. Prima, Towards a better comprehension of similarity measures used in medical image registration, Proceedings MICCAI, Lecture Notes in Computer Science, Springer, Berlin, 1999, pp. 555-566.

[35] R. Rojas, Neural Networks - A Systematic Introduction, Springer, Berlin, 1996.

[36] D.E. Rumelhart, G.E. Hinton, R.J. Williams, Learning internal representations by error propagation, in: D.E. Rumelhart, J.L. McClelland et al. (Eds.), Parallel Distributed Processing, Vol. 1, MIT Press, Cambridge, MA, 1986 (Chapter 8).

[37] D.E. Rumelhart, G.E. Hinton, R.J. Williams, Learning representations by backpropagation errors, Nature 323 (1986) 533-536. 
[38] N. Suzuki, A. Hattori, S. Kai et al., Surgical planning system for soft tissues using virtual reality, in: K.S. Morgan (Ed.), Medicine Meets Virtual Reality: Global Healthcare Grid, Studies in Health Technology and Informatics, Vol. 39, IOS Press, Amsterdam, 1997, pp. $159-163$.

[39] G. Székely, M. Bajka, Ch. Brechbühler et al., Virtual reality based surgery simulation for endoscopic gynaecology, Proceedings of Medicine Meets Virtual Reality 7, IOS Press, Amsterdam, 1999, pp. 351-357.

[40] D. Terzopoulos, K. Fleischer, Deformable models, The Visual Computer, Vol. 4, 1988, pp. 306-331

[41] K-H Wolf, J. Nuthmann, M. Plischke et al., Virtual endoscopic carpal tunnel release, Scientific Program of the RSNA, 1999.

[42] T.P. Yeh, J.M. Vance, Combining sensitive methods, finite element analysis, and free-form deformation to facilitate structural shape design in a virtual environment, Proceedings of the 23rd ASME Design Automation Conference, 1997.

[43] A. Radetzky, A. Nürnberger, D.P. Pretschner, Elastodynamic shape modeler: a tool for defining the deformation behavior of virtual tissues, Radiographics 20 (2000) 865-881.

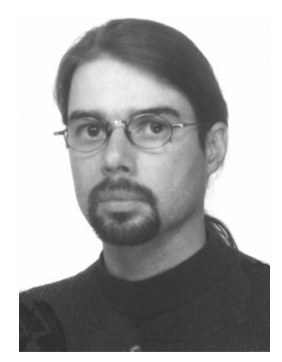

Andreas Nürnberger studied computer science and economics (operations research) at the University of Braunschweig, Germany, and graduated in computer science in 1996. Since 1997, he is working at the Otto-von-Guericke University of Magdeburg. His main research topics are neural networks, fuzzy systems and its combinations. He is escpecially interested in applications of these techniques in system identification, simulation and in the area of data mining. He is currently working on a Ph.D. thesis.

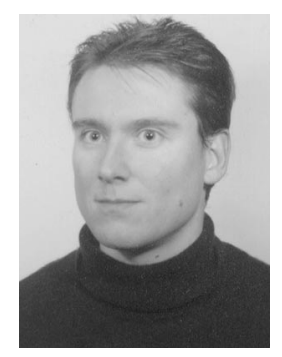

Arne Radetzky studied computer science at the University of Braunschweig, Germany, and graduated in 1996. Until 1999, he developed surgical simulators at the Institute of Medical Informatics at the University of Braunschweig. Now he is a Research Associate at the Department of Mechanical Engineering, Imperial College of Science, Technology and Medicine, London and leads the development of a simulation and planning system for neurosurgery at the Institute of Applied Sciences in Medicine, Salzburg, Austria.

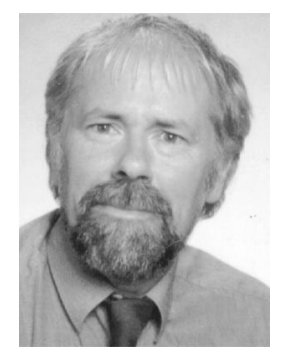

Rudolf Kruse studied mathematics and computer science at the University of Braunschweig, Germany, and graduated in mathematics in 1979. He received his Ph.D. in mathematics in 1980 and the venia legendi for mathematics in 1984 from the same university. From 1984 to 1986 he worked in an expert system research project of the Fraunhofer Society, and in 1986 he became professor for computer science at the University of Braunschweig, Germany. In 1996 he followed a call to the Otto-von-Guericke University of Magdeburg, where he is now a professor of Neural Networks and Fuzzy Systems. He has published and edited 16 books and is on the editorial board of four important international fuzzy systems journals. 